\title{
Laboreal
}

Volume $1 \mathbf{N}^{\circ} 1$ | 2005

Varia

\section{Envelhecimento, trabalho e cognição : 80 anos de investigação}

Envejecimiento, trabajo y cognición : 80 años de investigación

Vieillissement, travail et cognition : 80 ans de recherche

Ageing, work and cognition : 80 years of research

\section{Sara Ramos e Marianne Lacomblez}

\section{(2) OpenEdition}

Journals

Edição electrónica

URL: http://journals.openedition.org/laboreal/14201

DOI: $10.4000 /$ laboreal. 14201

ISSN: 1646-5237

\section{Editora}

Universidade do Porto

Refêrencia eletrónica

Sara Ramos e Marianne Lacomblez, «Envelhecimento, trabalho e cognição : 80 anos de investigação », Laboreal [Online], Volume $1 \mathrm{~N}^{0} 1$ | 2005, posto online no dia 01 dezembro 2005, consultado o 17 outubro 2019. URL : http://journals.openedition.org/laboreal/14201; DOI : 10.4000/ laboreal.14201

Este documento foi criado de forma automática no dia 17 outubro 2019.

Laboreal está licenciado com uma Licença Creative Commons - Atribuição-NãoComercial 4.0 Internacional. 


\section{Envelhecimento, trabalho e cognição : 80 anos de investigação}

Envejecimiento, trabajo y cognición : 80 años de investigación

Vieillissement, travail et cognition : 80 ans de recherche

Ageing, work and cognition : 80 years of research

Sara Ramos e Marianne Lacomblez

\section{NOTA DO EDITOR}

Manuscrito recebido em : Julho/2005

Aceite após peritagem em : Novembro/2005

\section{NOTA DO AUTOR}

Este artigo tem como base um capítulo da primeira versão da tese de Doutoramento em Psicologia de Sara Ramos, orientada por Marianne Lacomblez na Faculdade de Psicologia e de Ciências da Educação da Universidade do Porto, cuja conclusão está prevista para 2006.

\section{Evolução histórica do estudo do envelhecimento}

\subsection{Dos anos 20 ao pós-guerra : a emergência da problemática}

1 A história do estudo do envelhecimento é partilhada entre os primeiros trabalhos efectuados nos Estados Unidos e os realizados já na Europa no período do pós-guerra. É na década de 20 que surge a primeira preocupação com a temática do envelhecimento, através dos trabalhos de Miles na Universidade de Stanford na Califórnia, os quais consistiam em investigação fundamental sobre performances motoras e sobre a 
aprendizagem de trabalhadores do sector industrial (Welford, 1976). Ainda antes da guerra, em Inglaterra, foram efectuados alguns estudos acerca da influência do envelhecimento sobre a performance dos pilotos de caça da Royal Air Force, mas no final da guerra era o mundo industrial que colocava problemas de empregabilidade da mão-de-obra mais envelhecida.

2 É durante os anos 40 , num contexto de pós-guerra e de reconstrução económica, que se verifica, em Inglaterra, um interesse crescente pelo estudo do envelhecimento. Tal interesse deveuse às circunstâncias sócio-demográficas que caracterizavam essa época, ou seja, uma população envelhecida e o problema da reconversão dos militares para fazer face à falta de mão-deobra resultante das elevadas perdas humanas durante a II Guerra Mundial (sobretudo na população activa masculina), o que levou a considerar o potencial de produtividade de todos os trabalhadores disponíveis, incluindo os mais velhos (Teiger, 1995).

3 Em 1946 surge, em Cambridge, o primeiro laboratório dedicado aos problemas do envelhecimento, dirigido por Bartlett. Este laboratório, à semelhança dos trabalhos de Miles, centrava-se no estudo da performance humana com o objectivo de definir quais os tipos de trabalho industrial mais adequados para os trabalhadores menos jovens (Welford, 1976, 1986), ou seja, ambos os laboratórios estavam concentrados em avaliar o potencial dos mais velhos para o trabalho, abordando o tema de forma fundamental, isto é, medindo a performance em diferentes idades em tarefas de laboratório supostamente implicadas nas actividades industriais (Welford, 1976, 1985a). No entanto, se por um lado os objectivos eram semelhantes, os dois grandes pioneiros no estudo do envelhecimento diferiam quanto aos seus fundamentos teóricos. Nos Estados Unidos a equipa de Miles dedicava-se mais a estudos empíricos, enquanto a equipa de Cambridge utilizava sobretudo baterias de testes estandardizados que serviram de precursores para o desenvolvimento de importantes correntes teóricas dentro da própria ciência psicológica.

4 Aliás, a história do estudo do envelhecimento cruza-se, em diversos momentos, com a história da investigação em psicologia. Ao analisarmos a dimensão histórica estamos a analisar as condições externas globais no seio das quais de desenvolveram os fundamentos teóricos e metodológicos (Lacomblez, 1986) que orientaram a investigação. Entre 1940 e 1950 a pesquisa sobre o envelhecimento desenvolveu-se rapidamente e representa ainda hoje a primeira tentativa de construir um corpo teórico num campo largamente inexplorado (Welford, 1976), destacando-se em 1958 a publicação, pela Universidade de Cambridge, de uma das obras mais marcantes no domínio - "Ageing and human skill" (Welford, 1958). Em 1952 Bartlett retira-se do comando do laboratório de Cambridge e este sofre uma divisão em três grandes unidades, uma em Bristol dirigida por Murrell e dedicada à investigação experimental, a segunda em Liverpool direccionada para a investigação médica e uma terceira, em Londres, orientada para a investigação industrial e sob a direcção de Belbin.

5 No final dos anos 50, em Inglaterra, metade dos homens activos (entre os 20 e os 64 anos) tinham mais de 40 anos, verificandose, contudo, uma grande disparidade entre os diferentes sectores industriais, o que se assemelha genericamente à realidade actual dos países industrializados num contexto de envelhecimento demográfico global (Teiger, 1995), realidade que, quer nos anos 50 quer na actualidade, vem acentuar o interesse pela problemática. 


\subsection{Do final dos anos 50 aos anos $70:$ a aversão à investigação aplicada}

Segundo Welford e Teiger, o desenvolvimento da investigação sobre o envelhecimento parece atingir o seu pico precisamente no final da década de 50, iniciando-se, a partir daqui, uma trajectória marcada por um certo declínio que pode ser explicada por razões, uma vez mais, ligadas ao contexto sócio-demográfico da época. Nesta altura previa-se, em Inglaterra, um excesso de mão-de-obra para os anos 70, o que conduziu ao encorajamento da antecipação das reformas com vista a reduzir o desemprego (Welford, 1986). Além disso, assistia-se também a uma crescente aversão generalizada à investigação aplicada, a qual Welford (1986) explica recorrendo a diferentes causas : i) a conotação militar dos estudos realizados no período do pósguerra, sendo a investigação aplicada vista como negação da liberdade académica; ii) o facto de a investigação no terreno ser considerada por muitos investigadores mais árdua e menos elegante, exigindo competências de relacionamento interpessoal que nem todos partilham ; iii) a ênfase crescente na teoria, isto é, a insistência na verificação de hipóteses deduzidas de teorias pré-formadas mais do que em deixar a teoria emergir indutivamente dos factos.

O resultado disto ao nível metodológico foi uma alteração nas práticas iniciais em favor do estudo laboratorial de funções isoladas, tendência que deu origem a dois grandes desenvolvimentos, o uso generalizado dos testes psicométricos e dos questionários para comparar grupos de diferentes idades e o início dos estudos longitudinais (Welford, 1986). Mais uma vez, o percurso da investigação sobre o envelhecimento estabelece pontes evidentes com o desenvolvimento da psicologia e com os debates teóricometodológicos que caracterizaram a evolução e a história da disciplina.

8 Apesar da orientação para os processos fundamentais, a investigação ao longo dos trinta anos seguintes à segunda Grande Guerra é matizada por novas questões emergentes do domínio do trabalho e da recém surgida ergonomia (Marquié, 1993), alargando-se, progressivamente, ao resto da Europa. No final da década de 40, os estudos sobre o envelhecimento realizados em França centravam-se apenas nos aspectos demográficos mas, no princípio dos anos 60 , é organizado o primeiro colóquio CNRS (Centre National de Recherche Scientifique) consagrado ao envelhecimento.

No entanto, e tal como já foi referido, este é o período de eclipse em que o interesse social pelo envelhecimento se tornou marginal (Teiger, 1995) também em França. A problemática das relações idade-trabalho foi redescoberta mais tarde com as primeiras investigações em ergonomia no terreno (Teiger, 1995), impulsionadas por Wisner, Laville e Teiger. São, aliás, estes autores que editam, em França, a primeira recolha de trabalhos efectuados no domínio - "Âge et Contraintes de Travail: aspects sociologiques, psychologiques, physiologiques" (Laville, Teiger, \& Wisner, 1975).

\subsection{O final do século XX : a reinvenção da problemática}

10 O período de 1975 a 1990 é caracterizado por Teiger como o período do novo paradigma da velhice (Teiger, 1995). É nesta altura que o envelhecimento demográfico da população começa a instalar-se de forma mais evidente, a esperança de vida aumenta $\mathrm{e}$ a taxa de natalidade sofre decréscimos acentuados na maior parte dos países industrializados. 
11 Também o contexto sócio-económico se altera com os modelos de produção e de organização do trabalho em plena mutação. A evolução de um sistema industrial fordista/taylorista para o pósfordismo (Lacomblez, 2001) corresponde a novos modelos de concorrência internacional e favorecem a inovação e adaptação a novas modalidades de produção, com grandes repercussões para os trabalhadores (Teiger, 1995).

Uma destas repercussões é o desaparecimento da situação de pleno-emprego, talvez para sempre, o que se traduz numa alteração das carreiras profissionais, as quais se tornam cada vez mais flexíveis e descontínuas, alternando períodos de trabalho, formação e desemprego e pondo fim ao modelo clássico de ciclo de vida com três etapas sucessivas : formação, vida activa e reforma (Teiger, 1995).

13 Assiste-se ao progressivo abandono precoce da vida activa, ao aumento das chamadas pré-reformas, o que veio contribuir para que se alterasse a idade de referência habitualmente associada à velhice. Desta forma, criou-se uma nova categoria de trabalhadores "semi-envelhecidos" (entre os 45 e os 65 anos) que passaram a ser alvo de exclusão por determinadas políticas de emprego. Com este mecanismo as empresas conseguiram rejuvenescer as suas pirâmides etárias mas também se depararam com perdas ao nível do saber-fazer, da experiência e da cultura dificilmente reparáveis (Teiger, 1995).

14 Tal realidade leva-nos a questionar a evolução das representações sociais no que respeita à idade a partir da qual somos considerados "velhos", já que se trata mais de uma questão fundada não tanto em indicadores objectivos mas antes na dimensão cultural e nas práticas sociais específicas de cada época (Bourdelais, 1989). A noção de envelhecimento pode assim ser considerada uma criação relativamente recente ligada à sociedade industrial, uma "mistura de preconceitos e argumentos científicos" (Gaullier, 1988, p. 115, tradução livre) que traduz diferentes concepções do que se considera "trabalhador velho", em função da dinâmica sócio-económica: em período de crescimento ou de guerra, o trabalhador mais velho é valorizado porque se procura mantê-lo activo; em período de crise, pelo contrário, insiste-se numa representação associada ao absentismo, à sinistralidade, à doença e à falta de produtividade porque se procura fazê-lo sair do sistema (Gaullier cit in. Laville, 1989).

15 Todavia, este ressurgimento do interesse pela temática do envelhecimento relaciona-se igualmente com as profundas alterações verificadas no mundo do trabalho susceptíveis de terem produzido efeitos em termos de envelhecimento pelo trabalho. Assim, segundo os dados da Fundação Europeia para a Melhoria das Condições de Vida e de Trabalho, as últimas duas décadas têm sido marcadas por evoluções nem sempre favoráveis aos trabalhadores menos jovens, como é o caso do aumento da intensificação do trabalho. $\mathrm{O}$ agravamento dos constrangimentos temporais, da precariedade dos vínculos contratuais (Molinié, 2001a) e dos horários atípicos, as normas estritas e a curta margem de manobra deixada aos trabalhadores, que antes se encontravam sobretudo associados ao trabalho industrial, parecem ter-se generalizado a outras categorias profissionais (Molinié, 2003). Além disso, e segundo os mesmos dados, esta intensificação parece acompanhar-se de uma falta de investimento na formação e no desenvolvimento das competências (Molinié, 2003), penalizando de forma particular os trabalhadores mais velhos.

Desta forma, a conjuntura demográfica actual caracterizada pela baixa natalidade, pelo prolongamento da escolaridade, pela diminuição das medidas de reforma antecipada e, mais recentemente, pelo adiamento da idade da reforma, conduzindo ao 
envelhecimento progressivo da população activa, associada às evoluções das condições de trabalho, tornam necessária a intervenção nas situações de trabalho no sentido de preservar a saúde dos trabalhadores e garantir a sua eficácia (Molinié, 2001b), de modo a que estes se mantenham num estado de saúde satisfatório até à idade da reforma.

\section{Evoluções teóricas e conceptuais}

\subsection{Do biológico ao cognitivo}

17 A história da investigação sobre o envelhecimento é, assim, marcada por ressurgimentos e co-ocurrências entre acontecimentos históricos, económicos e demográficos (Teiger, 1995) que manifestam a interacção entre investigação e realidade social. Ao longo deste percurso, a abordagem científica do envelhecimento foi evoluindo à medida que o corpo teórico se desenvolveu.

Nos anos 50 as teorias do envelhecimento privilegiavam os aspectos biológicos (Teiger, 1995 ; Welford, 1980), como por exemplo a redução das células especializadas não susceptíveis de divisão como as do sistema nervoso; a progressiva rigidificação tecidular devido às proteínas de colagénio ; a limitação do número de divisões possíveis de células não especializadas; o enfraquecimento das defesas ao nível imunológico (Wisner, 1975).

19 Mais tarde, nos anos 80 , os estudos sobre o envelhecimento acabam por seguir o novo paradigma emergente na psicologia, orientando-se para a dimensão cognitiva. Este interesse renovado pelos aspectos cognitivos do envelhecimento situa-se no quadro do desenvolvimento global da corrente da psicologia cognitiva, a qual sofreu evoluções marcantes com o progresso da informática, colocando a ênfase nos processos de tratamento de informação (Teiger, 1995).

20 Ainda que as ciências cognitivas tenham dado os primeiros passos algumas décadas antes, nos anos 50 era ainda o behaviorismo que predominava dentro da psicologia, sendo nesta altura que alguns autores começam a distanciar-se deste paradigma (Dortier, 2002). É nos anos 70 que surge o termo "ciências cognitivas" e que o cognitivismo se assume como disciplina (Dortier, 2002), formando-se uma constelação de ciências que durante as décadas de 70 e 80 se desenvolveram de forma evidente. Consequentemente, a investigação passa a recorrer privilegiadamente a domínios como as neurociências e a neurobiologia que se encontravam em franca expansão com o estudo do funcionamento cerebral (Teiger, 1995; Varela, 1986), destacando-se os trabalhos de Birren (1960), Welford (1958; 1985b), Salthouse $(1989 ; 1994 ; 1996)$ e Pacaud $(1953 ; 1960 ; 1975)$. As

21 temáticas privilegiadas prendem-se agora com o armazenamento da informação (aprendizagem, memória imediata, memória diferida, memória de reconhecimento) a rapidez do tratamento da informação e as diferenças de performance com a idade em diferentes tarefas perceptivas e psicomotoras (tempos de reacção a estímulos visuais e sonoros, detecção de sinais, etc.).

\subsection{Da performance às estratégias :}

Todavia, este tipo de abordagem começa a ser posto em causa até mesmo por alguns destes autores. Em 1958, Welford tinha descrito o conceito de compensação, 
constatando que os mais velhos parecem usar diferentes métodos, compensando a perda de rapidez com um aumento da exactidão (Welford, 1986). Em 1978, surge também o uso do conceito de capacidade para realçar a noção de que a performance do sujeito será adequada enquanto a solicitação (demande) externa não esgotar a capacidade do indivíduo e que as capacidades máximas raramente são solicitadas pelas situações normais ao ponto de limitarem a performance (Welford, 1986).

Ainda nos anos 70 desenvolveu-se a noção de estratégia como elemento da performance, defendendo-se que não só as capacidades se modificam com a idade mas também o ajustamento das diferentes estratégias (Welford, 1980). O objecto de investigação deslocou-se então progressivamente da avaliação de performances à identificação de estratégias, do resultado aos processos subjacentes, do estudo das aptidões e capacidades ao reconhecimento da pluralidade de condutas, da variabilidade intra e inter-individual à diversidade de efeitos da idade sobre os vários aspectos da cognição, integrando as noções de plasticidade, compensação, regulação e vicariância (Teiger, 1995).

24 É este deslocamento do objecto de estudo que vai permitir o suporte teórico das diferenças observadas nos procedimentos operatórios em função da idade, quer nas tarefas de laboratório quer nas situações reais de trabalho, indo ao encontro da perspectiva da ergonomia e da psicologia do trabalho, uma vez que estas se interessam não tanto pelas capacidades fundamentais do ser humano mas antes pelas estratégias e modos operatórios dessas capacidades (Teiger, 1995), utilizados na situação real de uma actividade orientada para um fim.

É neste contexto que a noção de vicariância, desenvolvida por Reuchlin, assume particular relevo. Os processos vicariantes consistem em diferentes processos equifuncionais que, sendo substituíveis entre si, permitem ao indivíduo uma resposta adequada numa dada situação, o que significa renunciar a um modelo único do tratamento da informação e admitir a pluralidade de processos possíveis para uma mesma função cognitiva (Lautrey, 2002). Estes processos vieram colocar a ênfase na questão da variabilidade, a qual foi interpretada enquanto ruído ou erro de medida (Lautrey, 2002; Lautrey, Mazoyer, \& van Geert, 2002) durante a época do domínio experimentalista.

Reconhecer a influência destes processos e o estatuto da variabilidade enquanto propriedade intrínseca dos fenómenos em estudo (como foi o caso da psicologia diferencial) tem implicações metodológicas que fazem da própria variabilidade objecto de estudo. Aliás, é um facto globalmente descrito na literatura que a variabilidade tende a aumentar com a idade, quer ao nível intra-individual, já que o envelhecimento não afecta da mesma forma os diferentes tipos de capacidades, quer inter-individual (Millanvoye, 1995 ; Volkoff, Molinié, \& Jolivet, 2000 ; Welford, 1985b), encontrando-se, em alguns dos mais velhos, performances superiores a alguns dos mais novos, o que vem confirmar a impossibilidade de se estabelecer padrões ou índices de envelhecimento.

Contudo, as tentativas de estabelecer índices de envelhecimento resistem, ainda actualmente, aos argumentos dos estudos que acabamos de referir : a título de exemplo, podemos mencionar um instrumento largamente utilizado ao nível internacional, o Work Ability Index - WAI. Trata-se de um questionário curto ( 7 itens ) dirigido ao trabalhador, cujo objectivo é avaliar a adequação entre a sua aptidão e a exigência do trabalho, resultando um indicador quantitativo final que pode assumir quatro 
classificações : pobre, moderado, bom e excelente (de Zwart, Frings-Dresen, \& van Duivenbooden, 2002; Tuomi, Huuhtanen, Nykyri, \& Ilmarinen, 2001). A virtude essencial deste método reside na simplicidade da sua aplicação, sendo utilizado por alguns médicos do trabalho enquanto base de diálogo com o trabalhador, obviamente realizado ao abrigo do segredo profissional. Todavia, a facilidade de utilização do WAI apresenta também o risco de um uso afastado de preocupações deontológicas, susceptível de sustentar, sem fundamentos rigorosos, políticas de descriminação relativamente aos trabalhadores que obtêm uma avaliação mais fraca. Por outro lado, e do ponto de vista teórico, pela ausência de itens relativos aos condicionalismos do trabalho (só referidos pela apreciação da capacidade do trabalhador em assumi-los), o instrumento assenta numa lógica inversa à defendida pela ergonomia e pela psicologia do trabalho (Volkoff, 2005), já que coloca a ênfase na aptidão dos trabalhadores e nos seus recursos físicos e mentais em vez de orientar a intervenção para a situação de trabalho.

\subsection{Concepção desenvolvimental do envelhecimento}

Todas estas evoluções conduziram a uma nova concepção do envelhecimento. Não foi só a ênfase que se deslocou do biológico para o cognitivo, do declínio/deterioração para a compensação/plasticidade, da performance para as estratégias : é também a própria concepção do envelhecimento que se transforma.

A partir do momento em que o conceito de cognição se reajusta em torno das noções acima referidas (compensação, plasticidade, estratégia, regulação, vicariância), o que correspondeu a uma reestruturação interna do paradigma que dominava a psicologia o cognitivismo - também o envelhecimento passa a ser conceptualizado de forma desenvolvimental, processo que teve o seu início durante os anos 60 .

Se até aqui a idade adulta era considerada a idade de referência (Teiger, 1995) no que respeita à maturidade (comparando-se os mais velhos com os adultos jovens como se o desenvolvimento cessasse nesta idade), esta nova abordagem vem salientar a dimensão temporal do envelhecimento, isto é, o envelhecimento enquanto processo desenvolvimental que decorre desde o nascimento e ao longo de todo o ciclo vital e cujas características dependem da vivência subjectiva de cada indivíduo.

\section{Evoluções metodológicas}

31 Foi neste contexto de mudança que a forma tradicional de abordar esta problemática foi sendo alvo de duras críticas. A investigação experimental com tarefas de laboratório e o recurso privilegiado a técnicas fortemente associadas à lógica dos testes cognitivos foi sendo, progressivamente, posta em causa, inclusivamente por alguns autores que durante algum tempo partilharam esta abordagem. Este questionamento refere-se a diferentes parâmetros, os quais são discutidos nos pontos seguintes.

\subsection{População estudada}

As condições de amostragem encerram um dos principais limites apontados aos estudos de laboratório (Marquié, 1993). Efectivamente, os estudos sobre o envelhecimento ainda se encontram fortemente associados à gerontologia, focando-se em grupos etários 
extremos, ou seja, em comparações entre sujeitos adultos jovens e sujeitos adultos idosos, deixando de lado os períodos intermédios que permanecem menos bem conhecidos (Marquié, 1993) e que correspondem precisamente ao período de vida profissionalmente activo.

Por outro lado, o tipo de participantes englobados é também objecto de crítica, uma vez que nem todas as classes se encontram representadas nos estudos. Aliás, tal como refere Marquié, "o funcionamento cognitivo que melhor se conhece é, sem dúvida, o do estudante de psicologia" (Marquié, 1993, p. 67, tradução livre), já que os estudantes são largamente utilizados nos estudos experimentais, realizados em contexto académico. Desta forma, estamos a usar como referência uma amostra demasiado homogénea quer ao nível etário quer social, ao partilharem um conjunto de características pessoais que põem em causa a sua representatividade.

É ainda de referir que os estudos experimentais raramente utilizam como participantes trabalhadores de diferentes níveis de qualificação, o que vem reforçar os limites à generalização dos seus resultados a outras categorias sociais. Além disso, e mesmo para a população activa, é de salientar que nem todos os grupos profissionais estão expostos aos mesmos factores de envelhecimento nem às mesmas condições de trabalho. Um trabalhador de uma determinada idade pode ser considerado demasiado velho para um certo tipo de actividade ou jovem numa outra profissão (Marquié, 1993 ; Teiger, 1989), donde, os estudos deveriam considerar os diferentes ritmos de envelhecimento.

\subsection{Foco na quantificação}

A utilização dos testes psicométricos acarretou algumas desvantagens para o decurso da investigação psicológica, desviando as experiências laboratoriais das questões teóricas para as análises estatísticas (Welford, 1986). De facto, o interesse pela quantificação coincide, historicamente, com o uso dos testes estatísticos (Welford, 1980).

Contudo, segundo Welford $(1980 ; 1986)$, os resultados das comparações estatísticas deveriam ser expressos mais em termos de relações do que propriamente em termos de diferenças significativas. Isto acaba por ter implicações na forma como os investigadores desenham a investigação e os protocolos experimentais, orientando a sua acção para a obtenção de resultados estatisticamente convincentes.

A questão central parece ser o facto de este tipo de tratamentos se basear em critérios arbitrários que são, por vezes, utilizados de forma abusiva pelos investigadores, cuja preocupação se direcciona mais para níveis de significância do que para o significado real das diferenças observadas.

Concretamente no que respeita ao envelhecimento, os declínios que geralmente são verificados ao nível das capacidades elementares (percepção, memória, etc.), contribuem para que os efeitos da idade sejam avaliados tão frequentemente de forma quantitativa (Volkoff et al., 2000) através de indicadores de performance bastante limitados, ao marginalizarem o papel da experiência, da construção de estratégias e as influências situacionais. 


\subsection{Influência do contexto} transponíveis para a situação real. Enquanto nos estudos experimentais é possível conceber protocolos que permitam analisar a evolução de certas funções elementares com a idade, controlando o grau de experiência ou familiaridade dos sujeitos em relação às tarefas propostas, o problema é bem mais complexo em situação real (Paumès, 1995).

As investigações experimentais apoiam-se em "provas" muito distintas dos estudos efectuados em contexto real : as tarefas a realizar não são familiares, a valorização da experiência é limitada ou mesmo impossível, o resultado a atingir não corresponde a nenhum projecto pessoal ou profissional e o confronto dos mais velhos com uma situação que não dominam pode ser mal vivenciado (Volkoff et al., 2000). Ou seja, toda a situação é estranha, isolada, descontextualizada, sem passado nem futuro, não possibilitando ao sujeito a mobilização de todo o seu potencial.

41 Tal como refere Murrell (autor que dirigiu a unidade de Bristol, acima referida, e que se dedicou à investigação experimental), (...) qualquer pessoa que leia os resultados dos estudos de laboratório pode facilmente acreditar que todas as pessoas que atingem os 50 anos se tornam indivíduos lentos, esquecidos, meio-cegos, meio-surdos, temendo ser de pouca utilidade na indústria. De facto, muitos homens e mulheres asseguram o seu trabalho com perfeita satisfação dos seus empregadores. Isto não quer dizer que os resultados experimentais sejam falsos. $O$ problema parece proveniente do uso feito em laboratório de sujeitos ingénuos na prática da capacidade particular que está a ser testada (Murrell cit in. Teiger, 1995, p. 57, tradução livre).

O carácter pouco familiar da situação experimental exige, assim, uma certa prudência quando se pretende transferir os resultados obtidos em laboratório para as situações vividas no quotidiano, correndo o risco de sobrestimar as diferenças entre grupos etários (Marquié, 1993).

Por outro lado, no que respeita às funções cognitivas, os declínios são muito mais estudados do que os processos de construção, pois são mais regulares e mais simples de demonstrar, o que reforça as representações negativas acerca das capacidades dos trabalhadores mais velhos (Laville, 1995). Além disso, os métodos utilizados para quantificar as diferenças de idade consistem frequentemente em provas de curta duração que exigem capacidades extremas (Laville, 1995; Marquié, 1993; Welford, 1986) e onde só a performance é retida como critério, ignorando o papel da experiência adquirida pelos indivíduos. 0 estudo dos efeitos da idade nos mecanismos fundamentais coloca o indivíduo em condições limite de funcionamento cognitivo e de recurso mínimo aos seus conhecimentos, daí o carácter pouco preditivo dos dados experimentais face às capacidades reais (Marquié, 1993) que os indivíduos actualizam no seu meio familiar. Consequentemente, o valor preditivo deste tipo de abordagem restringe-se às situações em que se verifica uma solicitação mínima da experiência e, ao mesmo tempo, uma elevada solicitação das capacidades de base (Marquié, 1993), o que não corresponde à generalidade das situações de trabalho.

As capacidades elementares de tratamento de informação são muito dependentes do estado funcional e da situação (Rogoff, 1984) e, por isso, susceptíveis de declinar com a idade (Volkoff et al., 2000). Contudo, é globalmente aceite na literatura que os declínios das capacidades funcionais relacionados com a idade são progressivos e moderados,

Laboreal, Volume $1 \mathrm{~N}^{\circ} 1$ | 2005 
situando-se sobretudo nas performances extremas, as quais são raramente solicitadas nas situações reais (Volkoff et al., 2000; Welford, 1986). A actividade de trabalho mobiliza não só as capacidades elementares implicadas na tarefa mas assenta igualmente sobre a experiência do indivíduo (Paumès, 1995), a qual é essencial para estabelecer a relação com a tarefa e desenvolver estratégias que optimizem a performance (Volkoff et al., 2000).

Como já foi referido, a diversidade inter-individual aumenta com a idade mas as dispersões são, em geral, bem mais extensas nas tarefas mais complexas e é em situação real que se revela a diversidade de actividades humanas e o papel construtor da experiência e da idade (Laville, 1995). As abordagens experimentais realizadas em laboratório reduzem a expressão desta diversidade, uma vez que procuram mostrar a invariância dos fenómenos (Laville, 1995) mais do que a variabilidade dos mesmos.

É hoje globalmente aceite que as evoluções relacionadas com a idade são muito sensíveis aos constrangimentos vividos ao longo do percurso profissional e que a exposição a determinadas condições, nomeadamente de trabalho, pode ter um efeito ainda mais importante do que a idade por si só (Volkoff et al., 2000). A natureza das provas experimentais constitui uma das principais limitações à aplicação dos resultados obtidos em laboratório a situações de vida quotidiana, uma vez que a abordagem experimental é naturalmente redutora e mais adequada à análise de funções isoladas (Marquié, 1993) e não à apreensão de comportamentos complexos e integrados como é o caso das situações de trabalho.

47 Assim, no âmbito profissional, a dimensão idade não constitui somente um indicador temporal mas integra toda a génese do indivíduo, o seu passado profissional e o seu saber-fazer adquirido (Paumès, 1995), a sua história e a sua experiência, que resultam não numa performance mais ou menos boa mas numa forma diferente de realizar a sua actividade. Aliás, as modificações qualitativas da actividade com a idade têm sido sublinhadas desde há algum tempo, mas o alargamento do seu estudo em situação real de trabalho é recente, assistindo-se ao desenvolvimento de investigações centradas na análise de diferentes modos operatórios entre jovens e experientes, sobre as alterações e não comparações de performance em condições estritas e pré-definidas (Laville, 1995). Estas abordagens, desenvolvidas no âmbito da psicologia do trabalho e da ergonomia, valorizam modelos teóricos que combinam processos quer de declínio quer de construção (Laville, 1995) associados ao avanço da idade e vêm enfatizar o papel da experiência e do contexto de realização da actividade.

\section{Perspectivas actuais para o estudo do envelhecimento}

Após a proposta de abordagem histórica apresentada nos pontos anteriores, é indiscutível reconhecer que o domínio das relações idade-trabalho, a história sócioeconómica e cultural e a história científica estão estreitamente interligados (Teiger, 1995), o que significa que o estado actual das pesquisas efectuadas nesta área não se encontra estagnado mas continua a evoluir em função da conjuntura social e científica. Por outro lado, o facto de se verificarem evoluções marcantes na forma de fazer investigação não significa que as tradições mais recentes venham substituir as mais antigas ou que estas se tenham extinguido. A realidade é que coexistem diversas correntes teóricas, as quais sustentam diferentes perspectivas metodológicas e 
alimentam o debate sobre a forma de investigar o envelhecimento. Desta forma, a análise do conjunto de limitações do ponto anterior não tem a pretensão de desvalorizar a psicologia experimental, já que, em certos domínios, a sua contribuição continua a ser pertinente. Estas reservas podem igualmente contribuir para um alargamento das metodologias de investigação no sentido de uma maior preocupação com a validade ecológica (Marquié, 1993), como é o caso das abordagens em situação real igualmente referidas no ponto anterior.

Actualmente, é crescente a referência à necessidade de uma perspectiva cada vez mais global em matéria de envelhecimento, progressivamente orientada para uma abertura a novos conceitos e relações, considerando a história pessoal dos indivíduos, a forte ligação entre condições de vida e condições de trabalho e entre trajectórias sociais e profissionais (DREES, DARES, \& POSTE, 2003). A par das transformações associadas ao envelhecimento biológico e cognitivo, o avanço na idade deve ser encarado como um "desenrolar" de um ciclo de vida familiar ao longo do qual vão sendo assumidos diferentes papéis sociais e onde a natureza e as exigências se transformam em cada etapa (Queinnec, Gadbois, \& Prêteur, 1995).

É neste sentido que a abordagem da ergonomia tem vindo a ser objecto de um certo questionamento interno, ao centrar-se apenas na situação de trabalho. De facto, e quando se trata do processo de envelhecimento em particular, é evidente que uma enorme diversidade de contextos interagem de forma complexa e integrada, incluindo também variáveis da trajectória individual dos sujeitos, as quais têm sido negligenciadas por grande parte dos estudos realizados neste domínio.

51 As investigações mais recentes têm vindo já a reflectir sobre a importância de alargar a perspectiva, hoje tradicional, da ergonomia e da psicologia do trabalho e incluir na démarche da investigação aspectos que remetem para a história pessoal e social dos indivíduos. Ao nível metodológico, uma abordagem deste tipo assenta, fundamentalmente, na reconstituição dos percursos profissionais a partir da experiência subjectiva de cada sujeito, isto é, trata-se de conhecer não apenas os empregos ou postos ocupados mas, antes, aceder à actividade e condições reais de trabalho (Thébaud-Mony, 2005; Thébaud-Mony et al., 2003), bem como à sua articulação com as outras dimensões da vida. Trata-se, assim, de uma metodologia que "implica uma abordagem do trabalho fundada sobre métodos qualitativos utilizados quer na sociologia (histórias de vida) quer na ergonomia e na psicologia do trabalho (análise da actividade e do "vivido" do trabalho), tendo como objectivo essencial o retorno do próprio trabalhador sobre a sua própria experiência" (Thébaud-Mony et al., 2003, p. 6, tradução livre).

BIBLIOGRAFIA

Birren, J. (1960, Abril). Capacités psychologiques et processus du vieillissement du système nerveux. Comunicação apresentada em Le vieillissement de fonctions psychologiques et psychophysiologiques Colloques Internationaux du CNRS, Paris, França. 
Bourdelais, P. (1989). Vieillissement de la population ou artefact statistique ? Gerontologie et Société, $49,22-32$.

de Zwart, B., Frings-Dresen, M., \& van Duivenbooden, J. (2002). Testretest reliability of the Work Ability Index questionnaire. Occupational Medicine, 52, 4, 177-181.

Dortier, J.-F. (2002). Histoire des sciences cognitives. Sciences Humaines, 35, 6-14.

DREES, DARES, \& POSTE. (2003). Appel à projets de recherche lancé par la MiRe-DREES, la DARES et la Mission Recherche de La Poste. Paris : Ministère des Affaires Sociales, du Travail et de la Solidarité et Ministère de la Santé, de la Famille et des Personnes Handicapées.

Gaullier, X. (1988). Qu'est-ce qu'un salarié agé ? Gerontologie et Societé, 45, 115-127.

Lacomblez, M. (1986). L'analyse des motivations de l'homme au travail : archéologie d'un paradigme dominant. Le travail en sociologie, 14, 91-128.

Lacomblez, M. (2001). Analyse du travail et élaboration des programmes de formation professionnelle. Relations Industrielles/Industrial Relations, 56, 3, 543-578.

Lautrey, J. (2002). Le statut de la variabilité entre les individus en psychologie cognitive. In Jacques Lautrey, Bernard Mazoyer e Paul van Geert (Eds.), Invariants et variabilités dans les sciences cognitives (pp. 103-121). Paris : Éditions de la Maison des sciences de l'homme.

Lautrey, J., Mazoyer, B., \& van Geert, P. (2002). Invariants et variabilités dans les sciences cognitives. Paris : Éditions de la Maison des sciences de l'homme.

Laville, A. (1989). Vieillissement et Travail. Le Travail Humain, 52, 1, 3-20.

Laville, A. (1995). Travail et âges, de la recherche à l'action. In Jean-Claude Marquié, Dominique Paumès e Serge Volkoff (Eds.), Le travail au fil de l'âge (pp. 441-450). Toulouse : Octares Éditions.

Laville, A., Teiger, C., \& Wisner, A. (1975). Âge et Contraintes de Travail. Aspects sociologiques, psychologiques, physiologiques. Jouy-en-Josas : N.E.B. Éditions Scientifiques.

Marquié, J.-C. (1993). Vieillissement cognitif, expérience, et contraintes de l'environnement. Perspectives théoriques et ergonomiques. Thèse Docteur d'État ès Sciences, Université Paul Sabatier, Toulouse.

Millanvoye, M. (1995). Le vieillissement de l'organisme avant 60 ans. In Jean-Claude Marquié, Dominique Paumès e Serge Volkoff (Eds.), Le travail au fil de l'âge (pp. 175-209). Toulouse : Octares Éditions.

Molinié, A.-F. (2001a). Introdution. Évolutions des exigences du travail et itinéraires des salariés vieillissants. In B. Cassou, C. Buisset, D. Brugère, P. Davezies, F. Derriennic, G. Desplanques, A. Laville, J-C. Marquié, A. Touranchet e S. Volkoff (Eds.), Travail, Santé, Vieillissement : Rélations et évolutions (pp. 13-29). Toulouse : Octares Éditions.

Molinié, A.-F. (2001b). Parcours de travail et fins de vie active dans différentes générations. Quatre Pages Centre d'Études de l'Emploi, 45, 1-4.

Molinié, A.-F. (2003). Âge et Conditions de Travail dans l'Union Européenne. Dublin : Fondation européenne pour l'amélioration des conditions de vie et de travail. Office des publications officielles des communautés européennes.

Pacaud, S. (1953). Le vieillissement des aptitudes : déclin des aptitudes en fonction de l'âge et du niveau d'instruction. Biotypologie, XIV, 3/4, 65-94. 
Pacaud, S. (1960, Abril). Intervariabilité du groupe en fonction de l'âge : le facteur social dans le déclin des aptitudes. Comunicação apresentada em Vieillissement des fonctions physiologiques et psychophysiologiques Colloques internationaux du CNRS, Paris, França.

Pacaud, S. (1975). Le travailleur vieillissant : quelques réflexions sur ses difficultés, mais aussi ses facilités d'adaptation au travail. In Antoine Laville, Catherine Teiger e Alain Wisner (Eds.), Âge et Contraintes de Travail. Aspects sociologiques, psychologiques, physiologiques. (pp. 115-179). Jouy-en-Josas : N.E.B. Éditions Scientifiques.

Paumès, D. (1995). L'expression du vieillissement au travail : présentation de deux études menées auprès de contrôleurs aériens. In JeanClaude Marquié, Dominique Paumès e Serge Volkoff (Eds.), Le travail au fil de l'âge (pp. 305-327). Toulouse : Octares Éditions.

Queinnec, Y., Gadbois, C., \& Prêteur, V. (1995). Souffrir de ses horaires de travail : poids de l'âge et histoire de vie. In J-C. Marquié, D. Paumès e S. Volkoff (Eds.), Le travail au fil de l'age (pp. 342-378). Toulouse : Éditions Octares.

Rogoff, B. (1984). Thinking and learning in social context. In B. Rogoff e J. Lave (Eds.), Everyday's cognition : its development in social context. Cambridge : Harvard University Press.

Salthouse, T. (1989). Ageing and skilled performance. In A. Colley e J. R. Beech (Eds.), Acquisition and performance of cognitive skills (pp. 247-264) : John Wiley and sons.

Salthouse, T. (1994). Age-related differences in basic cognitive processes. Experimental Aging Research, 20, 249-255.

Salthouse, T. (1996, Setembro). Implications of adult age differences in cognition for work performance. Comunicação apresentada em Work after 45 ?, Stockholm, Suécia.

Teiger, C. (1989). Travailleurs "vieillissants" et formation : gageure ou enjeu ? Actes du Colloque "Le vieillissement au travail, une question de jugement" IRAT, 31-32, 40-54.

Teiger, C. (1995). Penser les relations âge/travail au cours du temps. In Jean-Claude Marquié, Dominique Paumès e Serge Volkoff (Eds.), Le travail au fil de l'âge (pp. 15-72). Toulouse : Octares Éditions.

Thébaud-Mony, A. (2005). Inégalités des parcours, connaissance et reconnaissance des cancers professionnels. Recherche/action en Seine-Saint-Denis. Actes du Séminaire Vieillissement et Travail 2004. Les évolutions de la santé au cours de la vie professionnelle : altération, préservation, construction., 141-158.

Thébaud-Mony, A., et al. (2003). Parcours-travail et cancers professionnels. Recherche-action en Seine Saint Denis France. PISTES, 5, 1, 1-28.

Tuomi, K., Huuhtanen, P., Nykyri, E., \& Ilmarinen, J. (2001). Promotion of work ability, the quality of work and retirement. Occupational Medicine, 51, 5, 318-324.

Varela, F. (1986). Connaître les sciences cognitives, Tendances et Perspectives. Paris : Éditions du Seuil.

Volkoff, S. (2005). Des comptes à rendre : usages des analyses quantitatives en santé au travail pour l'ergonomie. In Serge Volkoff (Ed.), L'ergonomie et les chiffres de la santé au travail : ressources, tensions et pièges (pp. 3-74). Toulouse : Octares Éditions.

Volkoff, S., Molinié, A.-F., \& Jolivet, A. (2000). Efficaces à tout âge ? Vieillissement démographique et activités de travail. Paris : Dossier no 16 du Centre d'Études de l’Emploi.

Welford, A. (1958). Ageing and human skill. Oxford : Nuffield Foundation University Press. 
Welford, A. (1976). Thirty years of psychological research on age and work. Journal of Occupational Psychology, 49, 129-138.

Welford, A. (1980). Where do we go from here ? In L.W. Poon (Ed.), Aging in the 1980's (pp. 615-621). Washington: American Psychological Association.

Welford, A. (1985a). Age, work, health and retirement : some fundamental considerations. Comunicação apresentada em 14th Annual Educational and Scientific Meeting of the Canadian Association of Gerontology, Hamilton, Ontario.

Welford, A. (1985b). Changes of performance with age an overview. In N. Charness (Ed.), Ageing and human performance (pp. 333-370). New York : John Wiley \& sons.

Welford, A. (1986). Forty years of experimental psychology in relation to age : retrospect and prospect. Experimental Gerontology, 21, 469481.

Wisner, A. (1975). Les phénomènes biologiques du vieillissement et les capacités des travailleurs de 40-50 ans. In Antoine Laville, Catherine Teiger e Alain Wisner (Eds.), Âge et Contraintes de Travail. Aspects sociologiques, psychologiques, physiologiques (pp. 47-75). Jouyen-Josas : N.E.B. Éditions Scientifiques.

\section{RESUMOS}

Esta revisão temática tem por objectivo traçar um percurso possível para a história da investigação sobre envelhecimento e trabalho, descrevendo a evolução dos estudos efectuados neste domínio, desde os primeiros trabalhos realizados nos anos 20 e os seus desenvolvimentos durante o período do pósguerra até à actualidade. Pretende-se reflectir de forma crítica sobre o modo como esta evolução aconteceu, as relações com as condições sócio-económicas e demográficas que caracterizaram cada época e as implicações teórico-metodológicas que esta evolução produziu, quer na forma como é conceptualizado o envelhecimento, quer ao nível dos métodos privilegiados nas investigações. Ao nível das implicações teóricas, serão analisadas com mais detalhe as abordagens centradas na dimensão cognitiva do envelhecimento, questionando as limitações e os argumentos que conduziram à emergência de outras tradições mais orientadas para as influências contextuais e situacionais que valorizam a experiência dos sujeitos, bem como as singularidades da sua história individual. Estas abordagens correspondem a uma tradição metodológica que privilegia a investigação de terreno, realizada em contexto real, largamente desenvolvida no campo da psicologia do trabalho e da ergonomia. Na parte final do texto são ainda abordadas orientações teóricas mais recentes que abrem caminho à génese de um novo rumo para o estudo do envelhecimento na sua relação com o trabalho.

Esta revisión temática tiene por objetivo trazar un trayecto posible para la historia de la investigación sobre envejecimiento y trabajo, describiendo la evolución de los estudios efectuados en este dominio, desde los primeros trabajos realizados en los años 20 y sus desarrollos durante el período de la posguerra hasta la actualidad. Se pretende reflexionar de forma crítica sobre la forma como esta evolución ha ocurrido, las relaciones con las condiciones socioeconómicas y demográficas que han caracterizado cada época y las implicaciones teóricometodológicas que esta evolución ha provocado, tanto en la forma como es conceptualizado el envejecimiento, como al nivel de los métodos privilegiados en las investigaciones. Al nivel de las implicaciones teóricas, se analizarán con más detalle los abordajes centrados en la dimensión cognitiva del envejecimiento, cuestionando las limitaciones y los argumentos que han conducido a la emergencia de otras tradiciones más orientadas hacia las influencias contextuales y de situación que valoran la experiencia de los sujetos, así como las singularidades de su historia 
individual. Estos abordajes corresponden a una tradición metodológica que privilegia la investigación de terreno, realizada en contexto real, largamente desarrollada en el campo de la psicología laboral y de la ergonomía. En la parte final del texto son también abordadas orientaciones teóricas más recientes que abren camino a la génesis de un nuevo rumbo para el estudio del envejecimiento en su relación con el trabajo.

Cette révision thématique a pour objectif de dresser un parcours possible pour l'histoire de la recherche sur vieillissement et travail, décrivant l'évolution des études effectuées dans ce domaine, depuis les premiers travaux réalisés dans les années 20 et leurs développements durant la période de l'aprèsguerre jusqu'à ce jour. On prétend mener une réflexion critique concernant le déroulement de cette évolution, ses relations avec les conditions socio-économiques et démographiques qui caractérisent chaque époque et ses implications théoricométhodologiques, tant sur le plan de la conceptualisation du vieillissement que sur celui des méthodes privilégiées dans les recherches. Au niveau des enjeux théoriques, on analysera plus particulièrement l'importance des approches centrées sur la dimension cognitive du vieillissement, en questionnant leurs limites ainsi que les arguments qui ont conduit à l'émergence d'autres traditions plus orientées vers les influences du contexte et de la situation, valorisant l'expérience des sujets ainsi que les singularités de leur histoire individuelle. Ces approches correspondent à une tradition méthodologique qui privilégie la recherche de terrain, réalisée en situation réelle, largement développée dans le champ de la psychologie du travail et l'ergonomie. La partie finale du texte fait référence à des orientations théoriques plus récentes qui ouvrent une voie nouvelle à l'étude du vieillissement dans sa relation avec le travail.

The aim of this thematic revision is to draw a possible course for the research history in ageing and work, describing the evolution of studies in this area, since the initial research in the 20 's and their development during the post-war period until today. We attempt to critically reflect on how this evolution occurred; its relations with socio-economic and demographic conditions that characterize each period; and the theoretical and methodological consequences of that evolution, either in the conceptualization of ageing or in research methods that are privileged. In the context of theoretical repercussions the approaches based on the cognitive dimension of ageing will be analysed in more detail, discussing the limits and the arguments that were conducive to the emergence of other traditions more directed towards the influences of context and situation, emphasizing the experience and the individual history of subjects. These approaches correspond to a methodological tradition witch privilege field research, conducted in real contexts, largely developed in work psychology and ergonomic domains. The final part of this text refers to the most recent theoretical orientations which lead to the genesis of a new approach to the study of ageing in their relation with work.

\section{ÍNDICE}

Palavras-chave: Envelhecimento, trabalho, cognição, história, metodologias

Mots-clés: Vieillissement, travail, cognition, histoire, méthodologies

Palabras claves: Envejecimiento, trabajo, cognición, historia, metodologías

Keywords: Ageing, work, cognition, history, methodologies 


\section{AUTORES}

\section{SARA RAMOS}

Centro de Psicologia da Universidade do Porto -Faculdade de Psicologia e de Ciências da Educação da Universidade do Porto Rua do Campo Alegre, 1055, 4169-004 Porto, Portugal scramos@mail.telepac.pt

\section{MARIANNE LACOMBLEZ}

Centro de Psicologia da Universidade do Porto -Faculdade de Psicologia e de Ciências da Educação da Universidade do Porto Rua do Campo Alegre, 1055, 4169-004 Porto, Portugal lacomb@fpce.up.pt 